\title{
Do educational interventions stop dating violence?
}

Vishal Bhavsar

COMMENTARY ON... COCHRANE CORNER ${ }^{\dagger}$

\begin{abstract}
SUMMARY
Violence is a critical challenge for society and it disproportionately affects young people. Violence experienced in an intimate relationship is associated with attempted suicide, depression and post-traumatic stress disorder, as well as poorer physical health. Interventions to limit intimate partner violence, especially in adolescents and young people, are a priority. This commentary examines a systematic review and meta-analysis of educational interventions for relationship and dating violence in young people aged $12-25$ years. Random-effects meta-analysis revealed a small statistical effect on knowledge, but no statistical associations with reduced violence. None of the included studies assessed health outcomes. The reviewers recommend further investigation of educational interventions in low- and middle-income settings, and studies with longer follow-up.
\end{abstract}

\section{DECLARATION OF INTEREST}

None.

\section{KEYWORDS}

Interpersonal relations; randomised controlled trial; violence; adolescent; childhood experience.

Preventing violence is a key part of improving health and quality of life, and may be especially relevant in young people. Quantitative research on violence prevention has tended to focus on specific forms of violence, such as bullying, childhood abuse, gang violence, workplace violence and intimate partner violence. Intimate partner violence involves the perpetration, or intended perpetration, of physical, sexual, psychological or financial harm to a partner (García-Moreno 2005).

Globally, intimate partner violence is reported by approximately 1 in 3 women and it may be more prevalent in low- and middle-income countries (García-Moreno 2005). Although intimate partner violence is common, evidence also indicates widespread underreporting. There is variation in the occurrence of intimate partner violence between countries and according to age and socioeconomic position (Foshee 2011). It is more common in young women than young men, and appears to cluster within individuals - that is, it is more common in those who have previously experienced it (Cotter 2016) and among those who have experienced other forms of violence, such as abuse during childhood (Finkelhor 2007). Although similar numbers of men and women report intimate partner violence in some surveys, these surveys have tended to collect information on instances of physical injury arising from violence, leading some to argue that such approaches systematically underestimate the occurrence and impact of intimate partner violence towards women, which can also take psychological, emotional and financial forms.

\section{Dating and relationship violence}

Dating and relationship violence, defined in the literature as intimate partner violence involving adolescents, is experienced by two-thirds to threequarters of young women in their mid to late teens in England, compared with one-third to half of young men of the same age (Barter 2015). Recent data suggest that $25 \%$ of 13 - to 16 -year-old girls in the UK have experienced pushing, slapping, hitting or being held down by a partner (Barter 2009). Dating and relationship violence is associated with worse physical health - sexually transmitted infections, teenage pregnancy, substance use, cancer, coronary heart disease, attempted suicide (Silverman 2001), depression (Devries 2013) and post-traumatic stress disorder (Eshelman 2012) are all more common in those who have experienced relationship and dating violence (Glass 2003). Violent victimisation by a partner during pregnancy is accompanied by poorer maternal and neonatal health, including premature delivery, low birth weight and fetal death (Campbell 2002). Adolescents who experience relationship and dating violence appear to be more likely to be the victims of, or to perpetrate, partner violence later in life (Singh 2012).
Vishal Bhavsar, $\mathrm{PhD}$, is a consultant psychiatrist and postdoctoral researcher in women's mental health at the Institute of Psychiatry,

Psychology and Neuroscience, King's College London, UK.

Correspondence Dr Vishal Bhavsar, Section of Women's Mental Health, Department of Health Services and

Population Research, Institute of

Psychiatry, Psychology and

Neuroscience, King's College London, 16 De Crespigny Park, London SE5 8AF, UK

Email: vishal.2.bhavsar@kcl.ac.uk

First received 26 Nov 2018 Final revision 11 Mar 2019 Accepted 25 Mar 2019

\section{Copyright and usage} (C) The Royal College of Psychiatrists 2019.

${ }^{\dagger}$ See this issue. 


\section{Rationale for a Cochrane review}

Given the frequency of relationship and dating violence, and the associated burden of morbidity, prevention is an important goal for health research. The systematic review in this month's Cochrane Corner (Fellmeth 2013) focused on the evidence on educational and skills-based interventions aimed at young people aged 12-25 years. This is in contrast to previous reviews on this topic, that have either focused on adult populations or, in younger populations, considered violence as a whole, rather than relationship and dating violence specifically (e.g. Mytton 2006; Adi 2007). The one previous review that did focus on educational interventions for relationship and dating violence in young people included non-randomised studies and did not provide a quantitative summary of included studies.

Fellmeth et al's review included interventions that focused on providing participants with knowledge and skills targeted at preventing future violence; research on screening programmes offering referral to other agencies was excluded. The included interventions were located in schools, colleges, homes and community settings. The investigators proposed that educational interventions for adolescents and young adults in these settings could improve effective communication and deal more effectively with relationship disappointments and stressors, as well as positively affect self-esteem.

\section{Scope of the review}

The reviewers focused on individuals aged 12-25 and included randomised controlled trials (RCTs), cluster RCTs and quasi-randomised controlled trials (studies where allocation was based on quasi-random methods, such as allocation by date of birth, day of the week or alternation). Studies with any type of setting and comparison group were included. Any study in which it was not possible to identify the violence prevention component of the intervention, or the intervention was screening and referral (rather than an educational intervention), was excluded from this review. The included primary outcomes for the review were:

- reduction in the number of episodes of relationship and dating violence

- reduction in injuries resulting from relationship and dating violence

- self-reported improvement in mental well-being

- adverse events (such as increased relationship and dating violence).

Secondary outcomes included improvements in behaviour or knowledge regarding relationship and dating violence, access to help or services, increased protective skills, and the cost, time and acceptability of the intervention itself. Both published and unpublished work was eligible for inclusion in the review.

Screening and selection of studies for inclusion was done by two reviewers. Extraction and management of study data, and assessment of risk of bias, was performed in line with usual Cochrane Collaboration practice: the title and abstract of each returned study were screened to check that the study was eligible for inclusion in the review. If the investigators were unable to access the full text of articles, they contacted authors by email. The reviewers developed a specific data extraction tool for the review, entering the data into a statistical software package. Extracted data from each study included the population, age range, intervention characteristics and outcomes. Two reviewers separately used the Cochrane Collaboration's 'risk of bias' tool to classify each included study for possible bias. The domains of the tool are listed in Box 1.

\section{Results of the review}

\section{The literature sample}

The investigators identified 98 articles eligible for inclusion on the basis of their titles/abstracts, but 17 of these were subsequently excluded because they did not have available full texts. Of the remaining 81 articles, a further 40 were excluded on the basis of the predefined exclusion criteria (e.g. they were not RCTs or there was no control group). Of

BOX 1 The domains of the Cochrane Collaboration's 'risk of bias' tool

Sequence generation How was the random sequence for allocating participants to intervention or control conditions generated, and was this process described in sufficient detail to satisfy the reviewers that the groups are indeed comparable?

Allocation concealment How was the sequence for allocation of participants to either treatment condition kept secret?

Blinding (masking) How were participants and researchers prevented from finding out which condition participants had been allocated to, and how were those assessing the outcomes masked to allocation?

Incomplete outcome data How much outcome data was missing and were the reasons for any missing outcome data made clear in the paper?

Selective outcome reporting Is there any suggestion in the paper that the investigators might have left out some of the outcome data that they collected?

(Higgins 2011) 
the 41 articles that remained, there were 38 studies. Of these, 33 were entered into the meta-analysis and 5 were excluded. Of these 5 studies, one used nonparametric analyses, preventing their inclusion in the meta-analysis, one did not report statistical significance tests, one did not report the number of participants, and two did not report a statistical comparison between intervention and control arms.

\section{Study settings and interventions}

All the included studies were carried out in the USA, with the exception of one conducted in South Korea. Most studies were carried out in educational settings. Most interventions were aimed at a general audience within the setting rather than, for example, individuals at high risk for the outcome. Interventions were mainly educational in content, although a small number also provided selfdefence training. Only one study provided intervention on communication skills. Interventions were delivered over a wide range of time periods, ranging from a single session of $50 \mathrm{~min}$ to 18 weekly sessions over 4 months. Some of the studies included a control intervention involving education on an unrelated topic, such as cultural issues, sexual health and career development. The included studies did not report effects on injuries, well-being, adverse events or intervention-related characteristics, but did report effects on episodes of relationship and dating violence, changes in behaviour, improvements in access to services and the attainment of protective skills.

\section{Reported outcomes}

Of 17 studies reporting effects on episodes of relationship and dating violence, most used the Sexual Experiences Survey (SES) or the revised Conflict Tactics Scale (CTS2) to measure this. Overall, 8 of these studies had sufficient data to be meta-analysed. Among secondary outcomes, most studies evaluated changes in behaviour, knowledge and attitudes (although attitudes were not included among the pre-specified secondary outcomes for the review). Six studies examined behavioural change, 12 tested change in knowledge and 7 assessed improved preventive skills. Most studies evaluated short-term outcomes (less than 6 months after the start of the study).

\section{Study quality}

Levels of bias in the included studies were variable: 29 studies gave unclear information on how random sequences were generated in order to allocate participants to the intervention or the control state. Around the same number had unclear information on how this allocation was concealed from investigators and participants. Assessor masking (blinding) was incompletely described in 37 studies. Incomplete outcome data was generally well-described, and outcomes were fully described where these were available. Funnel plots that the reviewers performed on their meta-analyses involving 10 or more studies suggested little evidence of publication bias affecting knowledge of relationship violence and attitudes towards relationship and dating violence.

\section{The meta-analyses}

For the synthesis of studies addressing episodes of relationship and dating violence, the reviewers conducted two meta-analyses, one on categorical outcome studies and the other on studies employing a continuous outcome (Box 2). Heterogeneity for the categorical outcome meta-analysis was high, so a random-effects meta-analysis model was used. The pooled effect of the included interventions was 0.77 (a 23\% risk reduction), indicating poor statistical evidence for any effect of intervention on the outcome. The continuous measures meta-analysis

\section{BOX 2 Meta-analyses}

Meta-analyses are quantitative summaries of the research on a particular question (such as 'Is drug $x$ effective for condition $y$ ?').

A systematic search of the literature is done, to attempt to find all previous answers to the question.

The answers from the identified studies, which are usually in the form of estimates of the size of an association, are compiled into a single spreadsheet and summarised.

These estimates are then used to compile what is essentially a weighted average of the different estimates from different studies. There are different ways of choosing the weighting that is used, but usually weights are calculated on the basis of the size of the study.

'Fixed' and 'random' effects, in the context of meta-analyses, refer to the way in which the different estimates are summarised:

- in essence, fixed-effects meta-analyses assume that a single underlying effect, or quantity, is being estimated, and they do not give an indication of the extent to which the different studies may be estimating different underlying effects

- random-effects meta-analyses assume that the different studies are estimating different underlying effects, and provide an estimate of the overall difference between the study estimates, also known as heterogeneity. Heterogeneity is usually summarised in the form of a statistic such as $P$.

Most meta-analyses in the psychiatric research literature are random-effects meta-analyses. 
also revealed a small reduction in the outcome, but this was not statistically supported.

No studies examined reduced episodes of injuries as an outcome or reported adverse events. There was a small and not statistically significant improvement in attitudes when all relevant studies were pooled. There was a mean increase in knowledge in the 10 studies with data on this; however, there was evidence of important statistical variability between the studies. There was nearly no evidence of an effect on protective skills.

\section{Discussion}

This was the first systematic review on this topic. It identified a wide range of available educational interventions aimed at reducing relationship and dating violence in community and educational settings. However, meta-analysis of the effect of such interventions on episodes of relationship and dating violence, changes in behaviour, attitudes and knowledge, and protective skills revealed no statistically reliable effects, except for a small statistical effect on knowledge. This statistical effect disappeared when studies that had a moderate or high risk of selection bias were removed, suggesting that further studies of better quality are need to satisfactorily answer the study question. Quality of included studies was assessed and reported using a validated tool. Heterogeneity in estimated effects was assessed appropriately using random-effects meta-analysis.

No included studies assessed health outcomes, which the reviewers consider to be an important limitation to the published work. Assessing the frequency of relationship and dating violence is challenging and prone to limited and incomplete disclosure. All of the studies came from high-income countries - in fact, all but one came from the USA. With the exception of two studies, the included studies had follow-up periods of less than a year, leaving little evidence on longer-term effectiveness.

In the included studies there was unclear evidence of bias introduced by randomisation, concealment of allocation and masking of assessors. Although the review authors acknowledged that masking was impossible, they noted that most of the studies provided staff training and some provided scripts for intervention delivery or monitored the fidelity of interventions. Studies not doing this were considered to be at high risk of performance bias.

Although the vast majority of the studies used cluster-randomised designs, only one study took statistical account of this in the analysis. The review authors suggest that as a result, included studies may have overestimated the size of the effects of interventions that were included in the meta-analysis.

\section{Limitations of the review}

The review may have had some limitations. Despite the reviewers' efforts, studies may have been missed because authors with relevant data did not respond to correspondence. Full texts were not available for 17 eligible studies, so these were omitted from the meta-analyses. These studies may have reported relevant data, including data in the opposite direction of the pooled effects actually identified, representing publication bias not assessable on the basis of the meta-analysed studies. The inclusion of quasi-randomised, as well as randomised, studies may have introduced bias, although this was not evident when the quasi-randomised studies were excluded from the meta-analyses. On the basis of the stated aims of the review, criteria used to select articles appear to have been appropriate, although the chosen age range was not justified.

\section{Suggestions for future studies}

Methodological shortcomings were widespread in the studies, so larger, more methodologically sound studies with longer follow-up are required. Most of the included studies assessed changes in knowledge and attitudes as outcomes. The review authors suggest the need for more evidence on the relationship between these outcomes and reductions in relationship and dating violence, which were the primary outcomes for this review. Studies employing a cluster-randomised design should take account of the effect of clustering in their statistical analyses. There should be more work in low- and middleincome countries to evaluate interventions on relationship and dating violence, as the causes of such violence might differ greatly between cultural groups.

\section{References}

Adi Y, McMillan AS, Kiloran A, et al (2007) Systematic Review of the Effectiveness of Interventions to Promote Wellbeing in Primary Schools. Report 3: Universal Approaches with Focus on Prevention of Violence and Bullying. University of Warwick.

Barter C, McCarry M, Berridge D, et al (2009) Partner Exploitation and Violence in Teenage Intimate Relationships. NSPCC.

Barter C, Wood M, Aghtaie N, et al (2015) Safeguarding Teenage Intimate Relationships (STIR): Connecting Online and Offline Contexts and Risks. Briefing Paper 2: Incidence Rates and Impact of Experiencing Interpersonal Violence and Abuse in Young People's Relationships. STIR.

Campbell JC (2002) Health consequences of intimate partner violence. Lancet, 359: 1331-6.

Cotter J, Drake RJ, Yung AR (2016) Adulthood revictimization: looking beyond childhood trauma. Acta Psychiatrica Scandinavica, 134: 368.

Devries KM, Mak JY, Bacchus LJ, et al (2013) Intimate partner violence and incident depressive symptoms and suicide attempts: a systematic review of longitudinal studies. PLoS medicine, 10: e1001439. 
Eshelman L, Levendosky AA (2012) Dating violence: mental health consequences based on type of abuse. Violence and Victims, 27: 215-28.

Fellmeth GLT, Heffernan C, Nurse J, et al (2013) Educational and skills-based interventions for preventing relationship and dating violence in adolescents and young adults. Cochrane Database of Systematic Reviews, 6: CD004534 (doi: 10.1002/14651858.CD004534.pub3).

Finkelhor D, Ormrod RK, Turner HA (2007) Re-victimization patterns in a national longitudinal sample of children and youth. Child Abuse \& Neglect, 31: 479-502.

Foshee VA, McNaughton Reyes HL, Ennett ST, et al (2011) Risk and protective factors distinguishing profiles of adolescent peer and dating violence perpetration. Journal of Adolescent Health, 48: 344-50.

García-Moreno C, Jansen H, Ellsberg M, et al (2005) WHO Multi-Country Study on Women's Health and Domestic Violence against Women. World Health Organization.
Glass N. Fredland N, Campbell J, et al (2003) Adolescent dating violence: prevalence, risk factors, health outcomes, and implications for clinical practice. Journal of Obstetric, Gynecologic \& Neonatal Nursing, 32: 227-38. Higgins JPT, Altman DG, Gøtzsche PC, et al (2011) The Cochrane Collaboration's tool for assessing risk of bias in randomised trials. BMJ, 343: d5928.

Mytton JA, DiGuiseppi C, Gough D, et al (2006) School-based secondary prevention programmes for preventing violence. Cochrane Database of Systematic Reviews, 3: CD004606.

Silverman JG, Raj A, Mucci LA, et al (2001) Dating violence against adolescent girls and associated substance use, unhealthy weight control, sexual risk behavior, pregnancy, and suicidality. JAMA, 286: 572-9.

Singh V, Walton MA, Whiteside L, et al (2012) Dating violence, alcohol, and other drug use among adolescents and young adults. Alcoholism: Clinical and Experimental Research, 36: 139A. 